\title{
Characterization of anogenital distance and its relationship to fertility in lactating Holstein cows
}

\author{
M. Gobikrushanth, ${ }^{*}$ T. C. Bruinjé, ${ }^{*}$ M. G. Colazo,, S. T. Butler, $\ddagger$ and D. J. Ambrose ${ }^{*} \dagger^{1}$ \\ *Department of Agricultural, Food and Nutritional Science, University of Alberta, Edmonton, AB, Canada T6G 2P5 \\ †Livestock Research and Extension Branch, Alberta Agriculture and Forestry, Edmonton, AB, Canada T6H 5T6 \\ ‡Teagasc, Animal and Grassland Research and Innovation Centre, Moorepark, Fermoy, Co. Cork, Ireland P61 C996
}

\begin{abstract}
Anogenital distance (AGD) serves as a marker for prenatal androgenization, reproductive development, and fertility in humans and rodents. The primary objectives of this observational study in lactating dairy cows were to (1) characterize the distribution and variability of AGD, (2) determine the relationship among AGD and potential postnatal AGD determinants of age and height, and (3) evaluate the associations between AGD and pregnancy to first artificial insemination (P/ $\mathrm{AI}$ ) and cumulative pregnancy by $250 \mathrm{~d}$ in milk (DIM) within parity groups (first, second, and third+ parities). The secondary objective was to evaluate the association between AGD and testosterone concentrations. The AGD (mm), age (yr), and height at hip $(\mathrm{cm})$ at the time of AGD determination, and aforesaid reproductive outcomes were determined in 921 Holstein cows (first, second, and third + parity; $\mathrm{n}=360,256$, and 305, respectively). Plasma concentrations of testosterone were determined in a subset of 93 cows. Overall, AGD had a normal distribution and high variability [mean ( \pm standard deviation); $131.0 \pm 12.2 \mathrm{~mm}$ ], was weakly associated with cow age and height (coefficient of determination $=0.09$ and 0.04, respectively), and had an inverse relationship with $\mathrm{P} / \mathrm{AI}$ in first- and second-parity cows, but not in third + parity cows. For every $1 \mathrm{~mm}$ increase in AGD, the odds of P/AI decreased by 3.4 and $2.4 \%$ for first- and second-parity cows, respectively. The optimal AGD threshold to predict probability of P/AI was $127.1 \mathrm{~mm}$ for both first- (sensitivity: 66.4; specificity: $56.6 \%$ ) and second-parity cows (sensitivity: 46.0; specificity: $70.4 \%$ ). Accordingly, first- and second-parity cows were categorized into either short or long AGD ( $\leq$ or $>127.1 \mathrm{~mm}$ ), and associations with reproductive
\end{abstract}

Received April 16, 2017.

Accepted August 10, 2017.

${ }^{1}$ Corresponding author: divakar.ambrose@gov.ab.ca outcomes were evaluated. First-parity cows with long AGD had lower P/AI (30.9 vs. 53.6\%) and decreased likelihood (hazard ratio: 0.68) of pregnancy by 250 DIM than those with short AGD. Similarly, secondparity cows with long AGD had reduced P/AI (28.3 vs. $44.4 \%$ ) and a tendency for decreased likelihood (hazard ratio: 0.76 ) of pregnancy by 250 DIM than in cows with short AGD. The association between AGD and testosterone was weak and nonsignificant. In summary, AGD in Holstein cows was normally distributed, highly variable, and weakly associated with age and height. Besides, AGD had an inverse relationship with P/AI and cumulative pregnancy by 250 DIM in first- and second-parity cows; however, such a relationship was not evident in older (third+ parity) cows.

Key words: anogenital distance, age, height, fertility

\section{INTRODUCTION}

Anogenital distance (AGD) has been defined as the distance from the center of the anus to either the posterior fourchette (Salazar-Martinez et al., 2004) or the clitoris (Sathyanarayana et al., 2010) in females. The in utero development of the perineum and caudal migration of genital tubercle, relative to the anus, are androgen dependent in humans and rodents (Langman, 1975; Bowman et al., 2003). Therefore, the variation in AGD is a reflection of fetal androgen exposure during its reproductive programming window in those species (Macleod et al., 2010; Dean at al., 2012). In this regard, Mendiola et al. (2012) reported that AGD was normally distributed in a population of young women with high variability. Several other studies demonstrated that the AGD was approximately twice as long in males as in females (Salazar-Martinez et al., 2004; Swan, 2008; Thankamony et al., 2009; Macleod et al., 2010; Sathyanarayana et al., 2010). Hence, AGD is not only a biological indicator of prenatal androgenization, but also a sexually dimorphic trait that may be used to determine fetal sex during early pregnancy. 
Prenatal exposure to excess androgen in female fetuses leads to poor reproductive system development in utero, subsequently resulting in long AGD and poor postnatal fertility outcomes in rodents, rabbits, and humans (Zehr et al., 2001; Banszegi et al., 2012; Mendiola et al., 2012; Mira-Escolano et al., 2014a; Wu et al., 2017, respectively). The onset of puberty was delayed in female mice with long AGD (Zehr et al., 2001). Rabbit does with long AGD delivered fewer and lighter offspring, and had male-biased litters (Banszegi et al., 2012). Women with long AGD, presumably exposed prenatally to high androgen concentrations in utero, had increased numbers of ovarian follicles (Mendiola et al., 2012) and greater testosterone concentrations during the early follicular phase (Mira-Escolano et al., 2014a) compared with women with short AGD. Recently, Wu et al., (2017) reported that women with longer AGD were approximately 18 times more likely to develop polycystic ovarian syndrome, which is characterized by hyperandrogenism and anovulation, than those with shorter AGD. The placenta is the primary source of androgens in dams bearing female fetuses in dairy cows (Mongkonpunya et al., 1975). Maternal concentrations of testosterone (110 to $166 \mathrm{pg} / \mathrm{mL}$ ) and androstenedione (936 to $1,400 \mathrm{pg} / \mathrm{mL}$ ) during gestation were highly variable among individual cows bearing female fetuses (Gaiani et al., 1984). Thus, it is plausible that the high variability in in utero exposure of female bovine fetuses to androgens affects AGD and postnatal reproductive functions as reported in humans and rodents. In women, AGD was not associated with the postnatal determinants of age, height, and weight, but it was associated with body mass index (Mira-Escolano et al., 2014b; Wu et al., 2017). Moreover, in one study (MiraEscolano et al., 2014a), for each millimeter increase in AGD in women, testosterone concentration increased by $0.006 \mathrm{ng} / \mathrm{mL}$. Similar studies characterizing AGD and its associations with age, height, reproductive outcomes, and testosterone concentrations have not been conducted in dairy cows.

If an association exists between the simple morphologic measure of AGD and reproductive performance in dairy cows, AGD could become a new reproductive phenotype with potential for use in future genetic selection to augment fertility. Therefore, the primary objectives of this observational study were to (1) characterize the distribution and variability of AGD, (2) determine the relationship among AGD and potential postnatal AGD determinants of age and height, and (3) evaluate the associations between AGD and pregnancy to first AI (P/AI) and cumulative pregnancy by 250 DIM within parity groups (first, second, and third+). The second- ary objective was to evaluate the association between AGD and testosterone concentrations.

\section{MATERIALS AND METHODS}

\section{Animals and Management}

This study was conducted at the Dairy Research and Technology Centre of the University of Alberta and 3 commercial dairy herds located in Alberta, Canada. Animals were housed and cared for in accordance with the requirements of Canadian Council on Animal Care (2009). Cows were fed a TMR (primary ingredients were barley or corn silage, alfalfa silage, alfalfa hay, and concentrates) formulated according to NRC (2001) to meet the requirements of a $650-\mathrm{kg}$ lactating cow producing $45.0 \mathrm{~kg}$ of milk/d, and had ad libitum access to water. Whereas cows were subjected to presynchronization followed by Ovsynch (first AI) and Ovsynch only (second $+\mathrm{AI}$ ) in the university research herd and one of the commercial herds (timed AI; herds A and $\mathrm{B}$, respectively), cows were predominantly inseminated based on estrus detection in 2 of the commercial herds (insemination at detected estrus; herds C and D, respectively).

\section{Determination of Anogenital Distance, Age, Height, Milk Yield, and Reproductive Measures}

Anogenital distance was defined as the distance from the center of the anus to the base of the clitoris (Figure 1a), and was measured using a stainless-steel digital calipers (Procise, The Innovak Group, Montreal, QC, Canada). The age of the cow (yr) at the time of AGD measurement was calculated by subtracting the date of birth from the date of AGD determination. The height at hip (hereafter referred to as "height") was determined using a livestock measuring stick (Jeffers, Dothan, AL) from the ground to the top of the cow's back (above hook bones). The AGD and height were measured by 2 individuals, with one person always measuring AGD and the other person measuring height. Data on AGD were collected during a single visit to each herd. Anogenital distance and height measurements were obtained from 921 cows (mean \pm SD: $171 \pm 93$ DIM) that had no apparent perineal abnormalities such as inflamed or lacerated vulva as indicators of trauma at parturition, and that were later than 14 DIM at the time of AGD determination. Data on 305-d matureequivalent milk yield and reproductive measures $(\mathrm{P} /$ AI and pregnancy by 250 DIM) were retrieved for all cows using DairyComp 305 herd management software (CanWest DHI, Guelph, ON, Canada). 


\section{Blood Sampling and Determination of Plasma Concentrations of Testosterone}

Blood samples were collected in a subset of 93 cows (research herd only) from coccygeal blood vessel using evacuated Vacutainer tubes (Becton Dickinson and Company, Franklin Lakes, NJ) containing sodium heparin immediately before the second GnRH of Ovsynch during a presynchronization/Ovsynch timed AI protocol described elsewhere (Gobikrushanth et al., 2017). Samples were placed on ice upon collection, centrifuged at $1,500 \times g$ for $20 \mathrm{~min}$ at $4^{\circ} \mathrm{C}$, plasma harvested, and frozen at $-20^{\circ} \mathrm{C}$ until assayed. Plasma concentrations of testosterone were determined at Endocrine Lab Services, University of Saskatchewan, Saskatoon, SK, Canada, using a commercial solid-phase RIA kit (ImmuChem, MP Biomedicals, LLC, Orangeburg, NY). Procedures were carried out according to manufacturer's instructions (http://www.radmed.com .tr/usr_img/urunler/free_testo_mp.pdf) except that

(a)

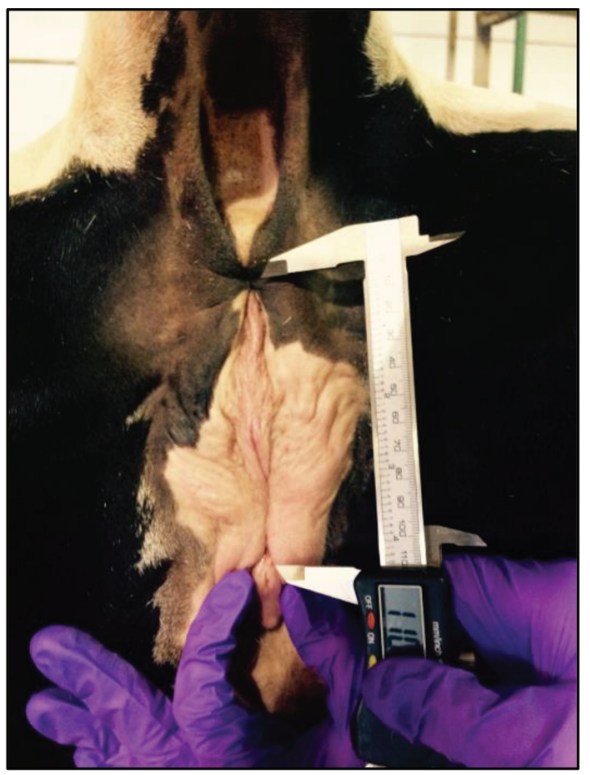

(b)

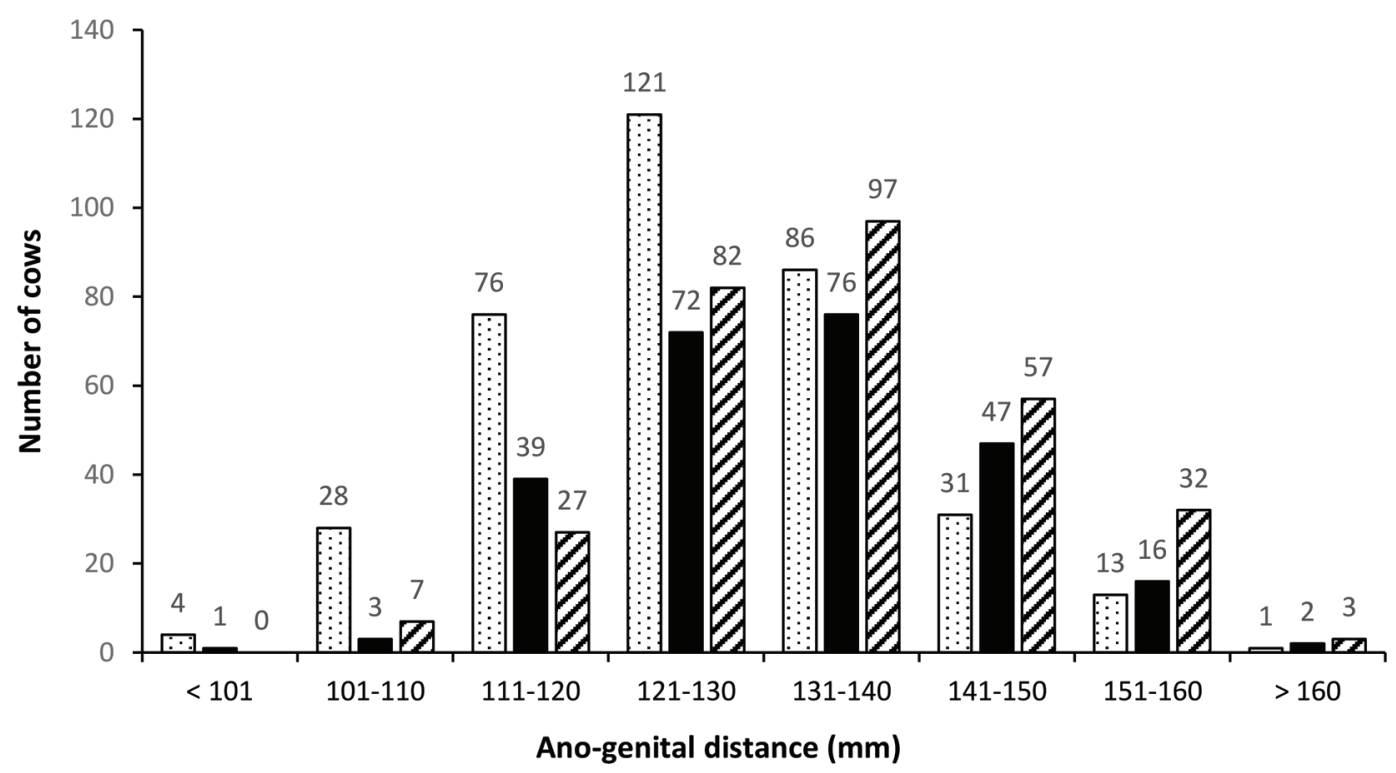

Figure 1. Positioning of digital calipers to measure anogenital distance (a): the distance from the center of the anus to the base of the clitoris. The distribution of anogenital distance (b) in first-parity (dotted bars; $\mathrm{n}=360$ ), second-parity (filled bars; $\mathrm{n}=256$ ), and third +-parity (hatched bars; $\mathrm{n}=305$ ) cows. Color version available online. 
standards were prepared by adding known quantities of testosterone to charcoal-stripped bovine serum, as the standards supplied with the kit were optimized for human samples. All samples were analyzed in a single assay; the intraassay coefficient of variation was $1.4 \%$ for low reference samples (mean, $4.2 \mathrm{pg} / \mathrm{mL}$ ) and $5.1 \%$ for high reference samples (mean, $8.8 \mathrm{pg} / \mathrm{mL}$ ).

\section{Statistical Analyses}

Data were analyzed using SAS version 9.4 (SAS Institute Inc., Cary, NC). Initially, AGD was determined by the same individual 3 times in 93 dairy cows from the university research herd, allowing sufficient time interval between measurements so that the subsequent readings were not influenced by the reader's memory (Salazar-Martinez et al., 2004). Later, the reliability and variability of AGD measurements were determined by evaluating the Pearson correlation coefficient (r) using CORR procedure and by evaluating the coefficient of variation using Excel 2016 (Microsoft Corporation, Redmond, WA), an approach similar to that of SalazarMartinez et al. (2004). The correlation between the 3 AGD measurements was high $(\mathrm{r}=0.98)$ and the coefficient of variation was low (1.1\%), allowing AGD to be determined only once by the same examiner in the rest of the animals. In addition, in the same subset of 93 cows from the university research herd, the association between AGD and plasma concentrations of testosterone was evaluated by linear regression analysis using REG procedure of SAS.

The descriptive statistics such as minimum, maximum, mean, and standard deviation (amount of dispersion indicative of variability) for AGD were determined using MEANS procedure of SAS for all cows ( $\mathrm{n}=$ 921) and separately for cows from first-, second-, and third+-parity groups $(\mathrm{n}=360,256$, and 305 , respectively). The differences in mean AGD between first-, second-, and third+-parity cows were tested using GLIMMIX procedure of SAS, where AGD was modeled against parity and the effect of herd was treated as random. The associations among age, height, and AGD were assessed by linear regression analysis using REG procedure of SAS.

The relationship between P/AI (binomial outcome) and AGD (predictor continuous variable) was first evaluated for each parity by logistic regression analysis using LOGISTIC procedure of SAS, and the estimated probabilities of $\mathrm{P} / \mathrm{AI}$ were plotted against AGD using Excel 2016. As the logistic regression model was nonsignificant for third+ parity, the rest of the analyses only focused on first- and second-parity cows.

The optimum threshold AGD that predicted the probability of $\mathrm{P} / \mathrm{AI}$, including specificity and sensitiv- ity, was determined using receiver operating characteristic curve analysis separately for first- and second-parity cows. The receiver operating characteristic curves analyze sensitivity and 1 - specificity. Sensitivity is the proportion of cows above the threshold that was diagnosed as pregnant to first AI, and specificity is the proportion of cows below the threshold and diagnosed as not pregnant to first AI. The threshold AGD was chosen based on the highest Youden's J statistic index. The significance of the threshold AGD was determined based on the area under the curve (AUC), where the AUC ranged from 0.50 to 1.00 , with AUC of 0.50 considered noninformative and the AUC of 1.00 considered perfect as previously described (Swets, 1988).

Thereafter, cows were categorized as either short or long AGD ( $\leq$ or $>$ threshold) based on the threshold AGD. The associations among categories of AGD (short and long AGD), herds (A, B, C, and D), type of AI (insemination at detected estrus and timed AI), 305-d mature-equivalent milk yield (low; Saverage and high; >average), and $\mathrm{P} / \mathrm{AI}$ were analyzed using the GLIMMIX procedure of SAS separately for first- and second-parity cows, whereas the model specifications included a binomial distribution and logit function, and an option to retrieve odds ratios. The P/AI was initially modeled against all of the aforementioned categorical variables and their interactions. As none of the interactions was significant, the final model only had the categorical variables modeled against P/AI.

The differences in intervals from calving to pregnancy between categories of AGD up to 250 DIM were evaluated using the Kaplan-Meier survival analysis (LIFETEST procedure), separately for first- and second-parity cows, and the results from Kaplan-Meier survival analysis were confirmed by a Cox proportional hazard model (PHREG procedure). Significant differences were reported if $P \leq 0.05$ and considered to be a tendency if $P>0.05$ and $\leq 0.10$.

\section{RESULTS AND DISCUSSION}

Anogenital distance has been identified as a marker of prenatal androgenization (Macleod et al., 2010; Dean at al., 2012) and associated with postnatal reproductive outcomes in female rats (Zehr et al., 2001), rabbits (Banszegi et al., 2012), and humans (Mendiola et al., 2012; Mira-Escolano et al., 2014a; Wu et al., 2017). To our knowledge, this is the first report to characterize the distribution and variation in AGD and evaluate its association with reproductive outcomes in lactating dairy cows. In the current study, AGD was normally distributed (Figure 1b) and highly variable [mean $( \pm \mathrm{SD}) ; 131.0 \pm 12.2 \mathrm{~mm}]$. The patterns of distributions and ranges reported (Table 1) remained approximately 
Table 1. Descriptive statistics for anogenital distance (AGD $)^{1}$ in lactating dairy cows

\begin{tabular}{lccc}
\hline Item & Minimum $(\mathrm{mm})$ & Maximum $(\mathrm{mm})$ & Mean $\pm \mathrm{SD}(\mathrm{mm})$ \\
\hline Overall AGD, all parities $(\mathrm{n}=921)$ & 96.0 & 170.0 & $131.0 \pm 12.2$ \\
AGD in first-parity cows $(\mathrm{n}=360)$ & 96.0 & 169.0 & $126.9 \pm 11.9$ \\
AGD in second-parity cows $(\mathrm{n}=256)$ & 100.0 & 170.0 & $132.5 \pm 11.7$ \\
AGD in third+-parity cows $(\mathrm{n}=305)$ & 103.0 & 164.0 & $134.5 \pm 11.4$ \\
\hline
\end{tabular}

${ }^{1} \mathrm{AGD}=$ the distance from the center of the anus to the base of the clitoris (Figure 1a).

the same within parities even though the mean AGD differed $(P<0.01)$ between first-, second-, and third+parity cows (126.9, 132.5, and $134.5 \mathrm{~mm}$, respectively). The distribution and variability of AGD found in dairy cows was comparable to the pattern of distribution and variability reported for AGD in women (Mendiola et al., 2012). In this regard, Mendiola et al. (2012) showed that AGD was normally distributed in a population of young women with a mean $( \pm \mathrm{SD})$ of $80.4 \pm 10.5 \mathrm{~mm}$. The normal distribution and the degree of dispersion for AGD in dairy cows and in women indicate that large phenotypic variation in AGD exists in different species.

The current study determined the phenotypic variation in AGD that was attributable to postnatal factors such as cow age and height. The overall associations between age and AGD $\left(\mathrm{R}^{2}=0.09 ; P<0.01\right.$; Figure $2 \mathrm{a})$ and between height and AGD $\left(\mathrm{R}^{2}=0.04 ; P<0.01\right.$; Figure $2 \mathrm{~b}$ ) were very weak when evaluated across all cows, and remained weak when height and AGD were evaluated separately for first-, second-, and third+parity cows (mean $\mathrm{R}^{2}=0.02 ; P<0.10$ ). In this regard, either weak or nonsignificant associations were reported between AGD and other anthropometric measures such as length and BW in female infants (Thankamony et al., 2009) and age, height, and weight in young women (Mendiola et al., 2012; Mira-Escolano et al., 2014b; Wu et al., 2017). Whereas Thankamony et al. (2009) reported weak associations between AGD and length $\left(\mathrm{R}^{2}=0.09\right)$, and between AGD and BW $\left(\mathrm{R}^{2}=0.03\right)$ in female infants at 24 mo of age, others (Mendiola et al., 2012; Mira-Escolano et al., 2014b; Wu et al., 2017) have shown that age, height, and weight were nonsignificant factors associated with AGD in women. However, MiraEscolano et al. (2014b) and Wu et al. (2017) reported a positive association between body mass index and AGD. Collectively, the findings in human studies and the current results in dairy cows suggest that AGD measures are largely independent of postnatal factors, and perhaps primarily influenced by the prenatal in utero concentrations of androgens as shown in rodents (Wolf et al., 2002; Hotchkiss et al., 2007; Dean et al., 2012).

Anogenital distance and $\mathrm{P} / \mathrm{AI}$ had a significant negative relationship in first- and second-parity cows
(Figure $3 \mathrm{a}$ and $3 \mathrm{~b}$, respectively); however, AGD was not associated with $\mathrm{P} / \mathrm{AI}$ in older cows $(P=0.30)$. In confinement dairy management systems such as those in Canada, the average longevity of a dairy cow is 2.5 lactations (about 5 yr of age), and only cows that excel in both fertility and milk production are likely to remain in the herd longer, which would at least partially explain the absence of association reported between AGD and fertility in third+ parity cows. Interestingly, the variation in AGD observed in third+
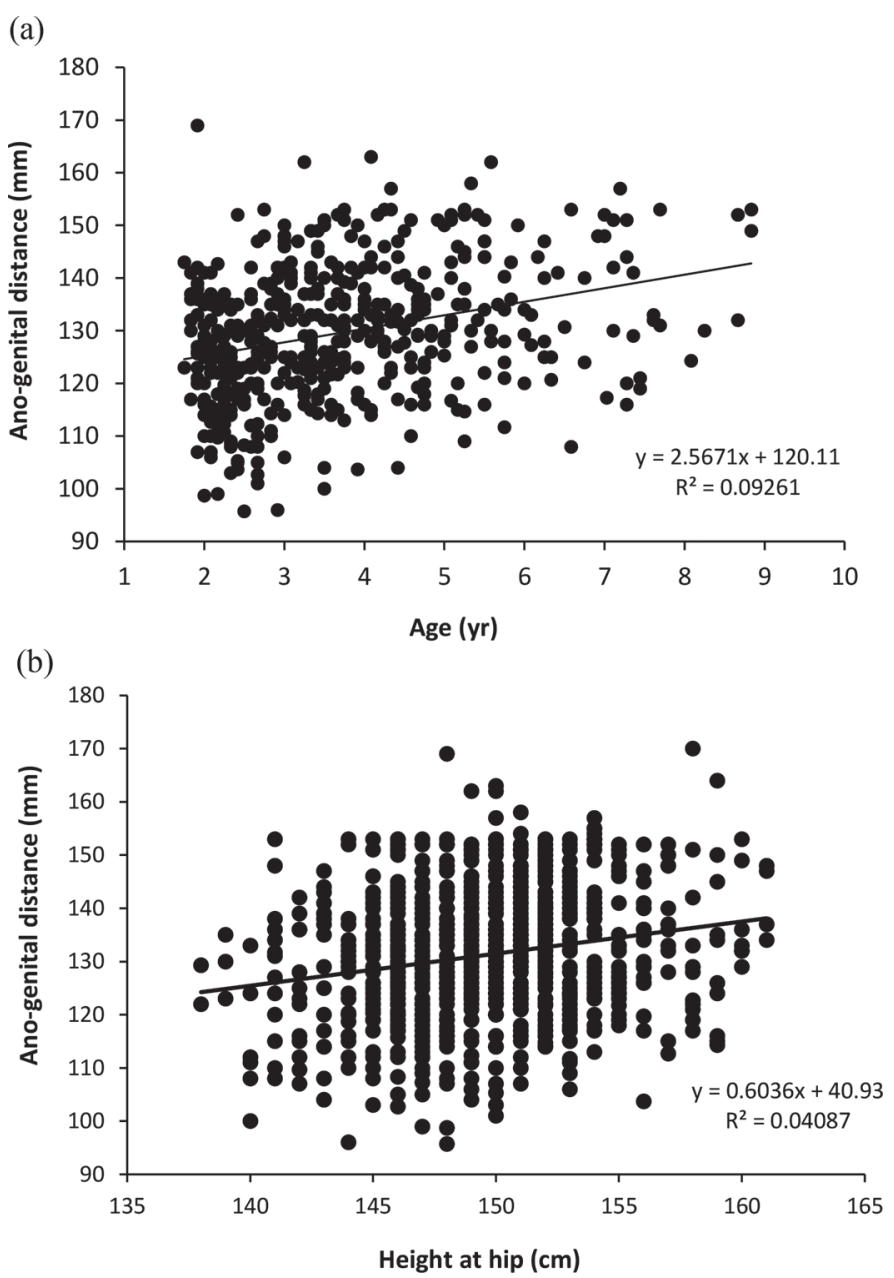

Figure 2. Association between age and anogenital distance $\left(\mathrm{a} ; \mathrm{R}^{2}\right.$ $=0.09 ; P<0.01)$ and height at hip and anogenital distance $\left(\mathrm{b} ; \mathrm{R}^{2}=\right.$ $0.04 ; P<0.01)$ in lactating dairy cows $(\mathrm{n}=921)$. 
parity cows was quite comparable to the variations in AGD for first- and second-parity cows. Hence, the lack of association between AGD and P/AI in third+ parity cows was not attributable to a more homogeneous AGD, but could be due to other potential factors that affect pregnancy establishment to first AI such as incidence of early postpartum diseases, negative energy balance, and higher milk production that are more common in older cows (Lee and Kim., 2006). Because the optimal AGD threshold to predict the probability of $\mathrm{P} / \mathrm{AI}$ was $127.1 \mathrm{~mm}$ for both first- and second-parity cows, only first- and second-parity cows were categorized into short or long AGD ( $\leq$ or $>127.1 \mathrm{~mm}$ ) groups and the associations with reproductive outcomes were evaluated. The sensitivity and specificity of the optimal AGD threshold that predicted $\mathrm{P} / \mathrm{AI}$ were moderate in both first- and second-parity cows (Figure $4 \mathrm{a}$ and $4 \mathrm{~b}$, respectively). We compared AGD groups separately for first- and second-parity cows because, in general, fertility declines as parity increases (Norman et al., 2009). Therefore, analyzing parity groups separately would
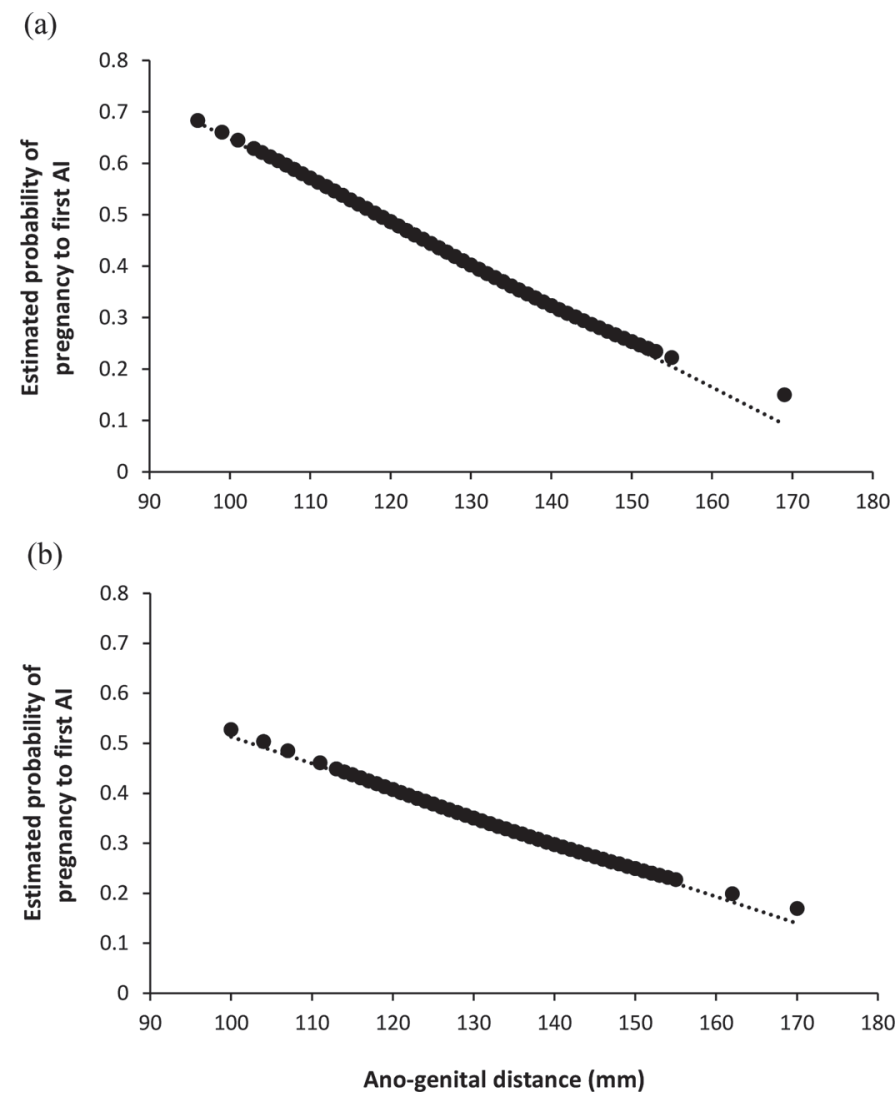

Figure 3. The estimated probability of pregnancy to first AI (P/ AI) plotted against anogenital distance (AGD) in first- and secondparity cows $(\mathrm{a} ; \mathrm{n}=360$ and $\mathrm{b} ; \mathrm{n}=256)$. For every 1 -unit $(\mathrm{mm})$ increase in AGD, the odds of conceiving to first AI decreased by 3.4 and $2.4 \%$ for first- and second-parity cows, respectively $(P<0.05)$. provide an insight into the true association between AGD and fertility within each parity.

Overall reproductive performance ( $\mathrm{P} / \mathrm{AI}$ and pregnancy by 250 DIM) was poorer in cows with long AGD than those with short AGD (Table 2 and Figure 5).

(a)

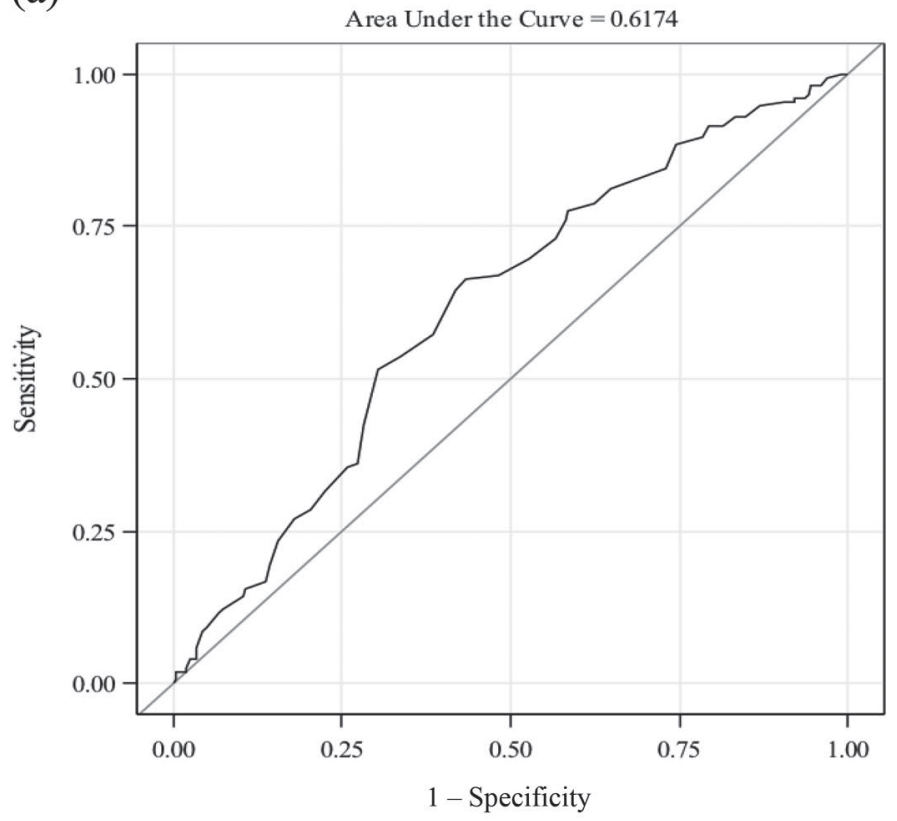

(b)

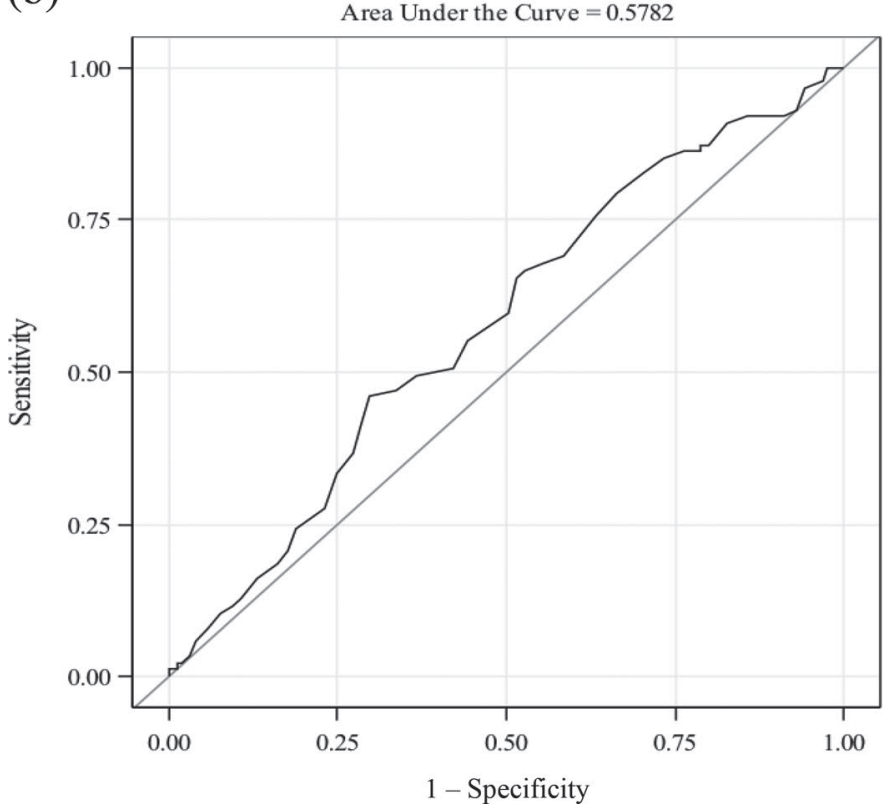

Figure 4. The receiver operating characteristic curve analysis for anogenital distance (AGD) that predicted probability of pregnancy to the first AI in first-parity ( $\mathrm{a} ; \mathrm{n}=360$; area under the curve: 0.62 ; sensitivity: 66.4 ; and specificity: $56.6 \% ; P<0.01$ ) and second-parity cows $(\mathrm{b} ; \mathrm{n}=256$; area under the curve: 0.58 ; sensitivity: 46.0 ; and specificity: $70.4 \% ; P=0.04)$. 
The results of the present study were broadly comparable to the outcomes reported for different AGD categories and fecundity/fertility in other species (Zehr et al., 2001; Banszegi et al., 2012; Mendiola et al., 2012; Mira-Escolano et al., 2014a; Wu et al., 2017). Specifically, the onset of puberty was delayed in female mice with long AGD (Zehr et al., 2001). Rabbit does with long AGD had smaller, lighter, and male-biased litters (Banszegi et al., 2012). Women with longer AGD had increased follicular recruitment and higher testosterone concentrations (Mendiola et al.,2012; Mira-Escolano et al., 2014a) during the early follicular phase, and were 18 times more prone to polycystic ovarian syndrome (Wu et al., 2017) than those with shorter AGD. Likewise, Steckler et al. (2005) showed that pregnant ewes treated twice weekly with testosterone propionate from 30 to $90 \mathrm{~d}$ of pregnancy had increased ovarian follicular recruitment in fetal ovaries. The authors (Steckler et al., 2005) suggested that this observation was due to activation of large number of primordial follicles into primary follicles by increasing the androgen receptor expression in primordial follicles as reported previously (Vendola et al., 1999).

We evaluated the association between AGD and testosterone in a subset of 93 cows, using an approach similar to that used by Mira-Escolano et al. (2014a) in women to determine whether cows with long AGD, presumably exposed to high androgens during fetal life, also had higher testosterone concentrations during postnatal life. In the current study, the association between AGD and testosterone was weak and nonsignificant $\left(\mathrm{R}^{2}=0.02 ; P=0.19\right)$, perhaps due to the relatively small sample size. In this regard, MiraEscolano et al. (2014a) reported that in women, testosterone concentration increased by $0.006 \mathrm{ng} / \mathrm{mL}$ for each $1 \mathrm{~mm}$ increase in AGD. Interestingly, a recent study (Mossa et al., 2010) that evaluated antral follicle counts (AFC) in dairy cattle showed that cows with high AFC $(\geq 25)$ had almost double the concentrations of circulating testosterone ( $\sim 60$ vs. $30 \mathrm{pg} / \mathrm{mL})$ throughout the

Table 2. Associations among anogenital distance (AGD) categories, herd, type of AI, 305-d mature-equivalent (MEQ) milk yield, and pregnancy to first AI in first- and second-parity dairy cows

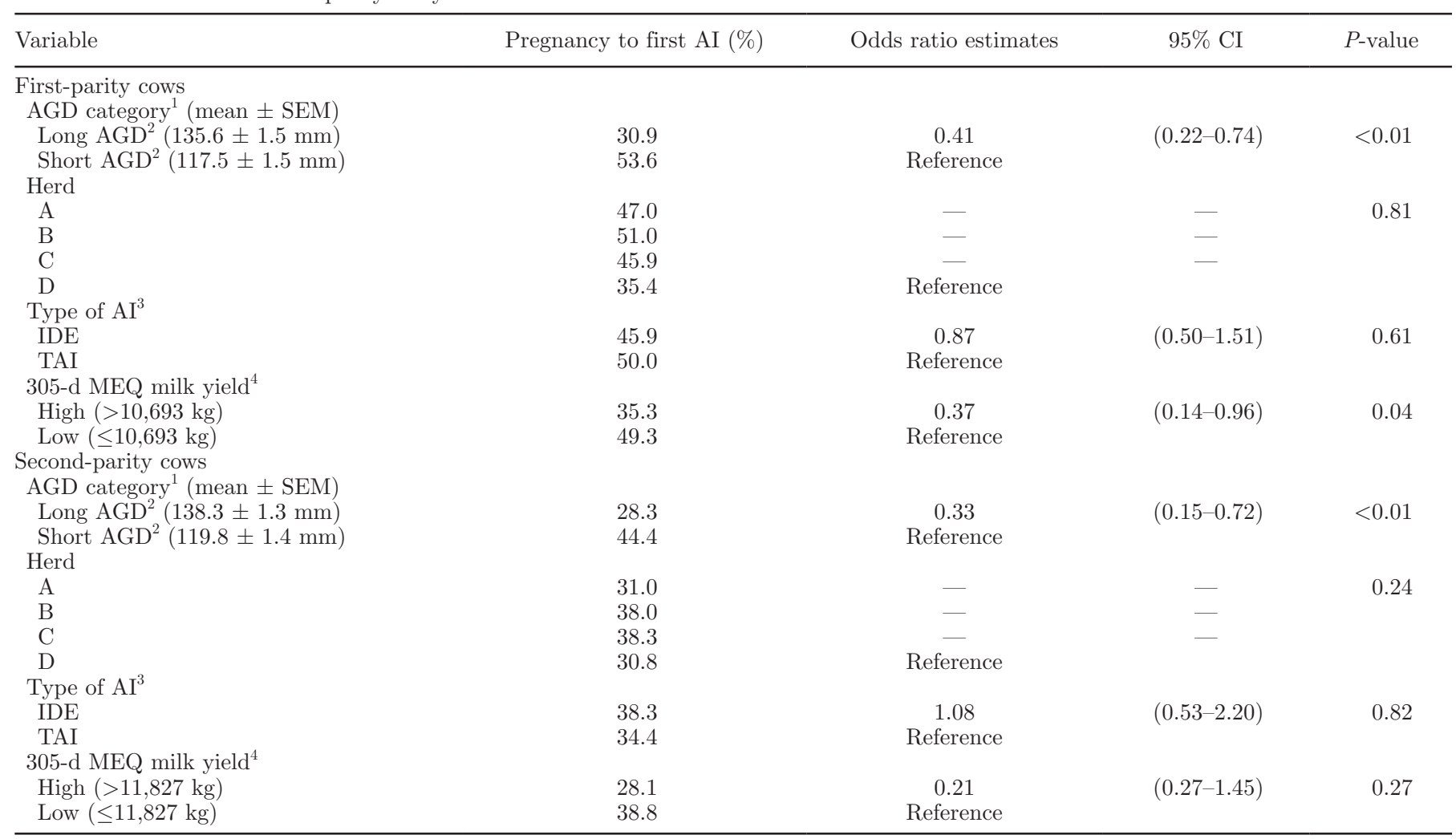

${ }^{1} \mathrm{AGD}=$ the distance from the center of the anus to the base of the clitoris (Figure 1a).

${ }^{2}$ AGD categories: cows that had their AGD $\leq$ or $>$ the threshold AGD (127.1 mm) that predicted probability of pregnancy for the first insemination in first- and second-parity cows were designated into either short- or long-AGD categories.

${ }^{3}$ Type of AI; cows were inseminated at detected estrus (IDE) or timed AI (TAI) following synchronization of ovulation.

${ }^{4} 305$-d MEQ milk yield categories were based on the average 305-d MEQ milk yield determined for first-parity (10,693 kg) and second-parity $(11,827 \mathrm{~kg})$ cows. 
estrous cycle compared with cows with low AFC $(\leq 15)$. In addition, dairy heifers with high AFC had poorer fertility and reduced longevity (Jimenez-Krassel et al., 2017). It is plausible that exposure of the female fetus to high levels of maternal androgens results in alterations in the development of female reproductive tissues including the external manifestation of long AGD, and these animals subsequently have increased follicular recruitment and androgen concentrations during estrous cycles that consequently lead to poor fertility outcomes and reduced longevity in dairy cattle. The proposed associations between maternal concentrations of androgens during the first trimester of pregnancy [under
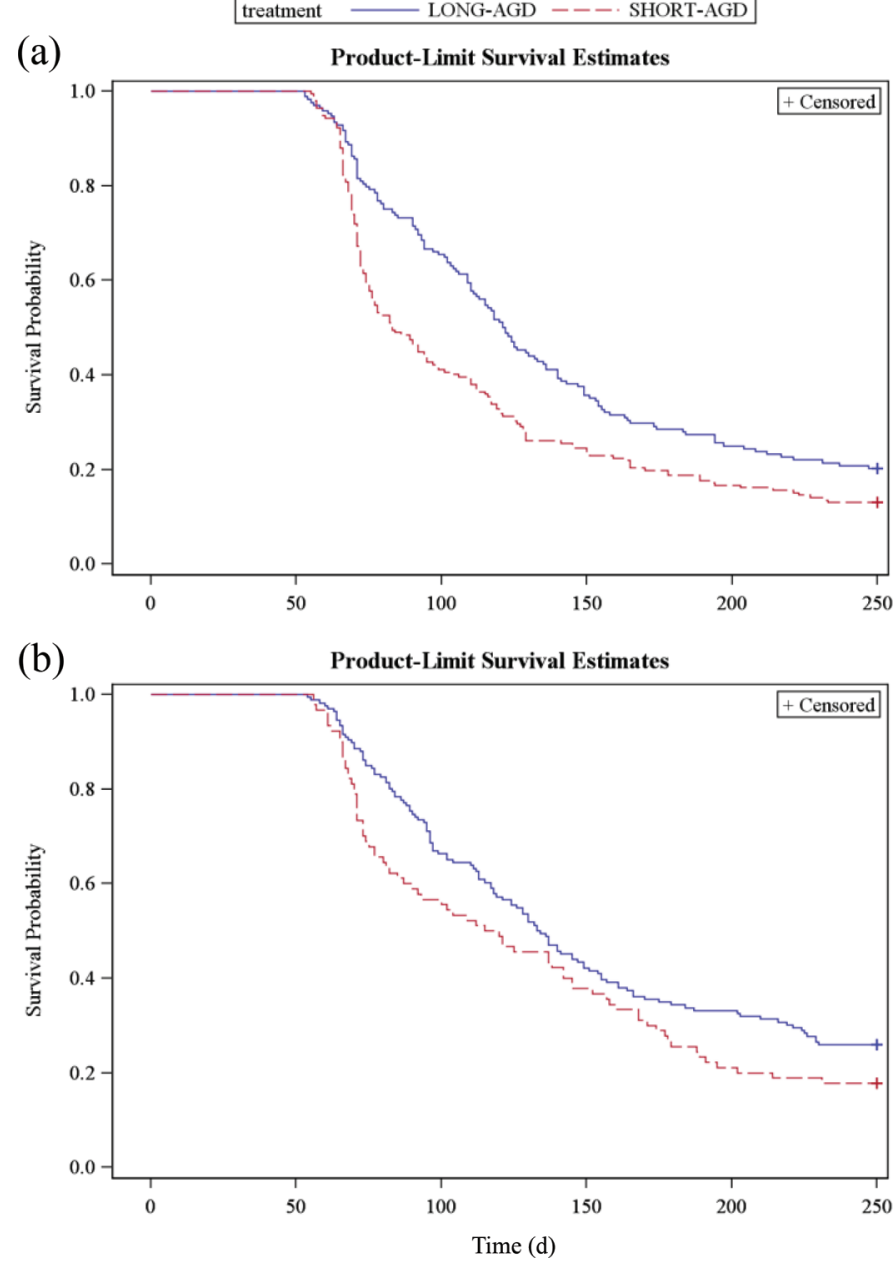

Figure 5. Kaplan-Meier survival curve that illustrates the proportion of cows [by anogenital distance (AGD) category] that remained nonpregnant by 250 DIM in first-parity (a) and second-parity (b) cows. The first-parity cows of long AGD $(135.6 \pm 1.5 \mathrm{~mm} ; \mathrm{n}=168)$ had significantly lower likelihood of pregnancy by 250 DIM (hazard ratio: $0.68 ; P<0.01)$ than cows of short AGD $(117.5 \pm 1.5 \mathrm{~mm} ; \mathrm{n}=$ 192). Similarly, the likelihood of pregnancy by 250 DIM tended to be lower (hazard ratio: $0.67 ; P=0.06$ ) for long AGD cows $(138.3 \pm 1.3$ $\mathrm{mm} ; \mathrm{n}=166)$ than those of short AGD $(119.8 \pm 1.4 \mathrm{~mm} ; \mathrm{n}=90)$ in the second parity. Color version available online. natural (endogenous) and experimental (exogenous) scenarios] and AGD at birth, puberty, and adulthood, and subsequent associations with fertility outcomes in dairy cows warrant further in depth investigations. The heritability of AGD and whether AGD could be used as a reproductive phenotype in genetic selection of dairy cattle to improve fertility also remain to be determined.

In conclusion, the present study has demonstrated for the first time that AGD is normally distributed and has high variability in lactating dairy cows. The variation identified for AGD was weakly associated with postnatal factors such as cow age and height. Furthermore, our results indicate an inverse relationship between AGD and P/AI and cumulative pregnancy by 250 DIM in first- and second-parity cows.

\section{ACKNOWLEDGMENTS}

This project was financially supported in part by Growing Forward 2, a federal-provincial-territorial initiative (Research Opportunities and InnovationInternal Initiatives Project \# DA7642064). M. Gobikrushanth is a recipient of the Teagasc-University of Alberta Walsh Fellowship sponsored by Alberta Innovates Biosolutions. The authors thank the staff of the Dairy Research and Technology Centre, University of Alberta, and the owners of the commercial dairy herds (Five Star Cattle Company, Lacombe, Alberta, Canada; Sunalta Farms Inc., Ponoka, Alberta, Canada; and Tuxedo Farms, Westlock, Alberta, Canada) for their assistance and cooperation.

\section{REFERENCES}

Banszegi, O., P. Szenczi, D. Kriszta, A. Bilko, and V. Altbacker. 2012. Anogenital distance as a predictor of attractiveness, litter size and sex ratio of rabbit does. Physiol. Behav. 105:1226-1230.

Bowman, C. J., N. J. Barlow, K. J. Turner, D. G. Wallace, and P. M. Foster. 2003. Effects of in utero exposure to finasteride on androgen-dependent reproductive development in the male rat. Toxicol. Sci. 74:393-406.

Canadian Council on Animal Care. 2009. Guidelines on the care and use of farm animals in research. teaching and testing. Accessed Sep. 6, 2017. http://ccac.ca/Documents/Standards/Guidelines/ Farm_Animals.pdf.

Dean, A., L. B. Smith, S. Macpherson, and R. M. Sharpe. 2012. The effect of dihydrotestosterone exposure during or prior to the masculinization programming window on reproductive development in male and female rats. Int. J. Androl. 35:330-339.

Gaiani, R., F. Chiesa, M. Mattioli, G. Nannetti, and G. Galeati. 1984. Androstenedione and testosterone concentrations in plasma and milk of the cow throughout pregnancy. J. Reprod. Fertil. 70:55-59.

Gobikrushanth, M., P. A. Dutra, T. C. Bruinje, M. G. Colazo, S. T Butler, and D. J. Ambrose. 2017. Characterization of the variability and repeatability of gonadotropin releasing hormone-induced luteinizing hormone responses in dairy cows within a synchronized ovulation protocol. J. Dairy Sci. 100:6753-6762. https://doi.org/ 10.3168 /jds.2016-12529.

Hotchkiss, A. K., C. S. Lambright, J. S. Ostby, L. Parks-Saldutti, J. G. Vandenbergh, and L. E. Gray Jr.. 2007. Prenatal testosterone 
exposure permanently masculinizes anogenital distance, nipple development, and reproductive tract morphology in female SpragueDawley rats. Toxicol. Sci. 96:335-345.

Jimenez-Krassel, F., D. Scheetz, L. Neuder, J. Pursley, and J. J. Ireland. 2017. A single ultrasound determination of $\geq 25 \mathrm{~mm}$ follicle $\geq 3 \mathrm{~mm}$ in diameter in dairy heifers is predictive of a reduced productive herd life. J. Dairy Sci. 100:5019-5027.

Langman, J. 1975. Medical Embryology: Human Development-Normal and Abnormal. 3rd ed. 160-200. The Williams \& Wilkins Company, Baltimore, MD.

Lee, J. Y., and I. H. Kim. 2006. Advancing parity is associated with high milk production at the cost of body condition and increased periparturient disorders in dairy herds. J. Vet. Sci. 7:161-166.

Macleod, D. J., R. M. Sharpe, M. Welsh, M. Fisken, M. Scott, G. R. Hutchison, A. J. Drake, and S. van den Driesche. 2010. Androgen action in the masculinization programming window and development of male reproductive organs. Int. J. Androl. 33:279-287.

Mendiola, J., M. Roca, L. Mı'nguez-Alarco'n, M. P. Mira-Escolano, J. J. Lo'pez-Espi'n, E. S. Barrett, S. H. Swan, and A. M. TorresCantero. 2012. Anogenital distance is related to ovarian follicular number in young Spanish women: A cross-sectional. Environ. Health 11:90.

Mira-Escolano, M. P., J. Mendiola, L. Mı'nguez-Alarco'n, M. Roca, A. Cutillas-Tolin, J. J. Lo'pez-Espı'n, and A. M. Torres-Cantero. 2014a. Longer anogenital distance is associated with higher testosterone levels in women: A cross-sectional study. BJOG 121:1359 1364.

Mira-Escolano, M. P., J. Mendiola, L. Minguez-Alarco'n, M. Roca, A. Cutillas-Tolin, J. J. Lo'pez-Espı'n, and A. M. Torres-Cantero. 2014b. Anogenital distance of women in relation to their mother's gynecological characteristics before or during pregnancy. Reprod. Biomed. Online 28:209-215.

Mongkonpunya, K., Y. C. Lin, P. A. Noden, W. D. Oxender, and H. D. Hafs. 1975. Androgens in the Bovine Fetus and Dam. Proc. Soc. Exp. Biol. Med. 148:489-493.

Mossa, F., F. Jimenez-Krassel, J. K. Folger, J. L. H. Ireland, G. W. Smith, P. Lonergan, A. C. O. Evans, and J. J. Ireland. 2010. Evidence that high variation in antral follicle count during follicular waves is linked to alterations in ovarian androgen production in cattle. Reproduction 140:713-720.
NRC. 2001. Nutrient Requirements of Dairy Cattle. 7th ed. Natl. Acad. Press, Washington, DC

Norman, H. D., J. R. Wright, S. M. Hubbard, R. H. Miller, and J. L. Hutchison. 2009. Reproductive status of Holstein and Jersey cows in the United States. J. Dairy Sci. 92:3517-3528.

Salazar-Martinez, E., P. Romano-Riquer, E. Yanez-Marquez, M. P. Longnecker, and M. Hernandez-Avila. 2004. Anogenital distance in human male and female newborns: A descriptive, cross-sectional study. Environ. Health 3:8.

Sathyanarayana, S., L. Beard, C. Zhou, and R. Grady. 2010. Measurement and correlates of ano-genital distance in healthy, newborn infants. Int. J. Androl. 33:317-323.

Steckler, T., J. Wang, F. F. Bartol, S. K. Roy, and V. Padmanabhan. 2005. Fetal programming: Prenatal testosterone treatment causes intrauterine growth retardation, reduces ovarian reserve and increases ovarian follicular recruitment. Endocrinology 146:31853193 .

Swan, S. H. 2008. Environmental phthalate exposure in relation to reproductive outcomes and other health endpoints in humans. Environ. Res. 108:177-184.

Swets, J. A. 1988. Measuring the accuracy of diagnostic systems. Science 240:1285-1293.

Thankamony, A., K. K. Ong, D. B. Dunger, C. L. Acerini, and L. A. Hughes. 2009. Anogenital distance from birth to 2 years: A population study. Environ. Health Perspect. 117:1786-1790.

Vendola, K., J. Zhou, J. Wang, O. A. Famuyiwa, M. Bievre, and C. A. Bondy. 1999. Androgens promote oocyte insulin-like growth factor I expression and initiation of follicle development in the primate ovary. Biol. Reprod. 61:353-357.

Wolf, C. J., A. Hotchkiss, J. S. Ostby, G. A. LeBlanc, and L. E. Gray Jr. 2002. Effects of prenatal testosterone propionate on the sexual development of male and female rats: A dose-response study. Toxicol. Sci. 65:71-86.

Wu, Y., G. Zhong, S. Chen, C. Zheng, D. Liao, and M. Xie. 2017. Polycystic ovary syndrome is associated with anogenital distance, a marker of prenatal androgen exposure. Hum. Reprod. 32:937-943.

Zehr, J. L., S. E. Gans, and M. K. McClintock. 2001. Variation in reproductive traits is associated with short anogenital distance in female rats. Dev. Psychobiol. 38:229-238. 